\title{
Correction to: Cross-Modality Imaging of Murine Tumor Vasculature-a Feasibility Study
}

Lydia M. Zopf, ${ }^{1}$ Patrick Heimel, ${ }^{2,9}$ Stefan H. Geyer, ${ }^{3}$ Anoop Kavirayani, ${ }^{1}$ Susanne Reier, ${ }^{1}$ Vanessa Fröhlich, ${ }^{4}$ Alexander Stiglbauer-Tscholakoff, ${ }^{4}$ Zhe Chen, ${ }^{5}$ Lukas Nics, ${ }^{5}$ Jelena Zinnanti, ${ }^{1}$ Wolfgang Drexler, ${ }^{5}$ Markus Mitterhauser, ${ }^{5,8}$ Thomas Helbich, ${ }^{4}$ Wolfgang J. Weninger, ${ }^{3}$ Paul Slezak, ${ }^{2}$ Anna Obenauf, ${ }^{6}$ Katja Bühler, ${ }^{7}$ Andreas Walter ${ }^{1}$

${ }^{1}$ Austrian BioImaging/CMI, Vienna BioCenter Core Facilities GmbH (VBCF), Vienna, Austria

${ }^{2}$ Ludwig Boltzmann Institute for Experimental and Clinical Traumatology in the AUVA Trauma Research Center, Austrian BioImaging/ CMI, Vienna, Austria

${ }^{3}$ Division of Anatomy, MIC, Medical University of Vienna, Austrian BioImaging/CMI, Vienna, Austria

${ }^{4}$ Department of Biomedical Imaging and Image-guided Therapy, Division of Molecular and Structural Preclinical Imaging, Medical University of Vienna, Vienna, Austria

${ }^{5}$ Medical University of Vienna, Vienna, Austria

${ }^{6}$ Research Institute of Molecular Pathology (IMP), Vienna Biocenter (VBC), Vienna, Austria

${ }^{7}$ VRVis Zentrum für Virtual Reality und Visualisierung Forschungs-GmbH, Austrian BioImaging/CMI, Vienna, Austria

${ }^{8}$ Ludwig Boltzmann Institute Applied Diagnostics, Vienna, Austria

${ }^{9}$ Core Facility Hard Tissue and Biomaterial Research, Karl Donath Laboratory, University Clinic of Dentistry, Medical University Vienna, Vienna, Austria

Correction to: Mol Imaging Biol https://doi.org/10.1007/s11307-021-01615-y

This article was updated to include Affiliation 9 for author Patrick Heimel, to add the Acknowledgments, and to correct the Supplementary Material.

Publisher's Note Springer Nature remains neutral with regard to jurisdictional claims in published maps and institutional affiliations. 